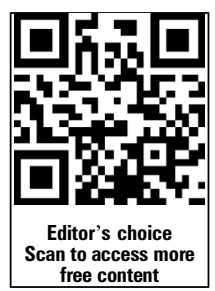

- Additional material is published online only. To view please visit the journal online (http://dx.doi.org/10.1136/jech2015-205848-)

${ }^{1}$ Department of Pediatrics, Children's Health Services Research, Indiana University School of Medicine, Indianapolis, Indiana, USA ${ }^{2}$ Department of Population Health Sciences, University of Wisconsin School of Medicine and Public Health, Madison,

Wisconsin, USA

${ }^{3}$ Division of Adolescent/Young

Adult Medicine, Boston

Children's Hospital and

Department of Pediatrics,

Harvard Medical School,

Boston, Massachusetts, USA

${ }^{4}$ Public Health Programs, College of Health Professions,

Ferris State University, Big

Rapids, Michigan, USA

${ }^{5}$ National Cancer Institute,

Bethesda, Maryland, USA

${ }^{6}$ Division of General Pediatrics

and Adolescent Health,

Department of Pediatrics,

University of Minnesota,

Minneapolis, Minnesota, USA

${ }^{7}$ Truven Health Analytics,

Durham, North Carolina, USA

\section{Correspondence to}

Dr Whitney P Witt, Center for Maternal and Child Health

Research, Truven Health

Analytics, 4819 Emperor

Boulevard, Suite 125, Durham,

NC 27703, USA; whitney.

witt@truvenhealth.com

Received 31 March 2015

Revised 10 September 2015

Accepted 23 September 2015

Published Online First

23 October 2015

\section{CrossMark}

To cite: Cheng ER, Park H,

Wisk LE, et al. J Epidemiol

Community Health

2016:70:245-252.

\title{
Examining the link between women's exposure to stressful life events prior to conception and infant and toddler health: the role of birth weight
}

\author{
Erika R Cheng, ${ }_{1}^{1}$ Hyojun Park, ${ }_{1}^{2}$ Lauren E Wisk, ${ }^{3}$ Kara C Mandell, ${ }^{2}$ Fathima Wakeel, ${ }^{4}$ \\ Kristin Litzelman, ${ }^{5}$ Debanjana Chatterjee, ${ }^{6}$ Whitney P Witt ${ }^{7}$
}

\begin{abstract}
Background The life course perspective suggests a pathway may exist among maternal exposure to stressful life events prior to conception (PSLES), infant birth weight and subsequent offspring health, whereby PSLEs are part of a 'chains-of-risk' that set children on a certain health pathway. No prior study has examined the link between PSLEs and offspring health in a nationally representative sample of US mothers and their children. We used longitudinal, nationally representative data to evaluate the relation between maternal exposure to PSLEs and subsequent measures of infant and toddler health, taking both maternal and obstetric characteristics into account.
\end{abstract}

Methods We examined 6900 mother-child dyads participating in 2 waves of the nationally representative Early Childhood Longitudinal Study-Birth Cohort $(n=6900)$. Infant and toddler health outcomes assessed at 9 and 24 months included overall health status, special healthcare needs and severe health conditions. Adjusted path analyses examined associations between PSLEs, birth weight and child health outcomes.

Results In adjusted analyses, PSLEs increased the risk for very low birth weight (VLBW, <1500 g), which, in turn, predicted poor health at both 9 and 24 months of age. Path analyses demonstrated that PSLEs had small indirect effects on children's subsequent health that operated through VLBW.

Conclusions Our analysis suggests a chains-of-risk model in which women's exposure to PSLEs increases the risk for giving birth to a VLBW infant, which, in turn, adversely affects infant and toddler health. Addressing women's preconception health may have important downstream benefits for their children, although more research is needed to replicate these findings.

\section{INTRODUCTION}

Despite advances in healthcare and implementation of social service and early intervention programmes, the prevalence of children with disabilities in the USA remains high and may even be increasing among some groups. ${ }^{1}$ Recent data from the National Health Interview Survey found that the prevalence of childhood disabilities rose $16 \%$ from 2001 to affect nearly six million US children in 2011, with notable increases observed for children with neurodevelopmental and mental health disorders. ${ }^{1}$ Understanding factors that influence these trends is essential to inform intervention efforts that seek to improve children's developmental and health trajectories.
The life course perspective conceptualises health as arising from cumulative effects of events that occur across the lifespan, as well as from intergenerational effects. ${ }^{2-6}$ Disparities in child health are thus consequences of both exposures before and during pregnancy and early life exposures. In a previous study, Witt et $a l^{7}$ found that a woman's exposure to stressful life events prior to conception (PSLEs) increased her risk for having a very low birth weight (VLBW) infant. In turn, a large body of literature demonstrates that low birth weight (LBW) contributes to infant and childhood morbidity. ${ }^{8-11}$ The life course perspective suggests a pathway may exist among PSLEs, birth weight and subsequent child health, whereby PLSEs are part of a 'chains-of-risk' that set children on a certain health pathway. In this scenario, PSLEs increase a woman's risk of having a VLBW baby, and VLBW, in turn, contributes to poor health in early childhood. ${ }^{6}$ Understanding the direct and/or indirect relationships between PSLEs and children's early health could elucidate mechanisms linking children's health to the preconception experiences of their mothers, and thus lend insight into how these 'chains-of-risk' might be interrupted.

Using a nationally representative sample of Danish women, Li et $a l^{12}$ found that maternal bereavement (eg, death of a parent, spouse or close relative) within 6 months prior to pregnancy was associated with attention deficit hyperactivity disorder (ADHD) and adolescent overweight ${ }^{12}$ in offspring. Class et $a l^{13}$ noted associations between women's preconception stress and infant mortality in a Swedish cohort. While these European-based studies are among the first to suggest that women's exposure to PSLEs may affect the immediate and long-term health of offspring, no study has examined the relation between PSLEs and obstetric and child health outcomes in a national cohort of US families.

We used longitudinal data from the nationally representative Early Childhood Longitudinal StudyBirth Cohort (ECLS-B) to evaluate maternal exposure to PSLEs and subsequent measures of infant and toddler health (overall health status, diagnoses of special healthcare needs (SHCNs) and the presence of severe health conditions), taking both maternal and obstetric characteristics into account.

\section{METHODS}

\section{Data source}

The ECLS-B is a nationally representative, longitudinal cohort study of nearly 10700 US live-born 
infants. The probability sample was drawn from the 4 million US children born in 2001, with oversampling of children from minority groups, twins, and children born at LBW and VLBW, based on registered births from the National Center for Health Statistics vital statistics system. The sampling frame excluded births to mothers under 15 years and children adopted or deceased before the initial collection wave. Data for this study were from the first two waves, which occurred when children were 9 and 24 months of age. ${ }^{14}$

Parents of participating children in the ECLS-B provided informed consent, and the data collection procedures were approved by National Center for Education Statistics (NCES) as ensuring confidentiality. We obtained a licence agreement with NCES for analysis of ECLS-B's restricted data. We rounded unweighted sample sizes to the nearest 50 to comply with NCES guidelines. ${ }^{14}$

Participants were eligible for this study if the survey respondent was the biological mother ( $\mathrm{n}=10550)$; we excluded 450 children missing birth certificate data. For each child sampled as a twin $(n=1500)$, we randomly selected one child from each pair to remain in the sample. Testing models with multiples removed did not influence our findings. Given the previously identified relationship between PSLEs and VLBW, ${ }^{7}$ we then restricted our sample to children born with VLBW $(<1500 \mathrm{~g})$ and normal birth weight (NBW; between 2500 and $3999 \mathrm{~g}$ ). Our final sample thus consisted of 6900 children and their mothers who participated in the second ECLS-B data wave and who had complete covariate information.

To evaluate the robustness of the results, we repeated analyses comparing children born LBW versus NBW (see online supplementary appendix 1) and included children born LBW and high birth weight (HBW; $\geq 4000 \mathrm{~g}$ ) with NBW children as the reference outcome (see online supplementary appendix 2).

\section{Measures}

Stressful life events prior to conception

Our approach to defining PSLEs is detailed elsewhere. ${ }^{7} 15$ Briefly, we derived the date of conception using birth certificate information on the length of gestation and the infant's date of birth. We coded women as having experienced a PSLE if they reported that one or more of the following events occurred prior to conception: (1) death of their mother, (2) death of their father, (3) death of a previous live-born child, (4) divorce (5) separation from partner (6) death of a spouse or (7) problems with infertility (see online supplementary appendix 3). These experiences are considered stressful life events and/or have been operationalised as such in previous research. ${ }^{16-19}$ In our sample, nearly $75 \%$ of women who experienced any PSLE had an event occur within 1 year prior to conception (data not shown).

\section{Very low birth weight}

Birth weight (continuous grams) was derived from the birth certificate. We used a cutpoint of $<1500 \mathrm{~g}$ to denote VLBW and the 2500-3999 g range to denote NBW.

\section{Infant and toddler health}

We examined three child health binary indicators, assessed by maternal report at 9 and 24 months: (1) overall health status, reported on a five-point Likert scale and dichotomised as 'excellent, very good, or good' versus 'fair or poor'; (2) a clinically diagnosed SHCN; and (3) any severe health condition, defined as asthma, respiratory illness, ear infection or any gastrointestinal-related illness that required an emergency room visit or overnight hospitalisation. SHCNs included blindness, difficulty seeing, difficulty hearing or deafness, problem with mobility or using legs, and heart defects. Additional SHCNs assessed at 9 months included diagnoses of cleft lip or palate, failure to thrive, problem with arms or hands, Down syndrome, Turner syndrome, Spina bifida, and any other special needs or limitations and at 24 months included diagnoses of developmental delay, epilepsy or seizures, mental retardation, lactose intolerance, and food allergies or sensitivities. Children whose mothers endorsed any of these conditions at either time point were considered to have SHCNs. As a sensitivity analysis, we excluded genetic disorders/chromosomal abnormalities from our definition of SHCN; results were consistent with the main findings.

\section{Maternal health, stress and sociodemographic factors}

Birth certificate data were used to determine if women had experienced any pregnancy complication (eg, anaemia, eclampsia) or had a chronic condition (eg, cardiac disease, chronic hypertension). At 9 months, self-reported maternal health was coded on a five-point Likert scale ranging from 1 (excellent) to 5 (poor). Pre-pregnancy body mass index (BMI) was calculated from selfreported height and weight prior to pregnancy (dichotomised as overweight or obese BMI $\geq 25$ vs normal or underweight BMI $<25$ or unknown). We evaluated timing of initiation of prenatal care (first trimester; second or third trimester; or no prenatal care), whether the child was a singleton or multiple, and the number of prior live births/parity ( 0 vs $\geq 1)$. Women were coded as having experienced a stressful life event during pregnancy if they indicated that the death of a close relative, divorce or partner separation occurred during their pregnancy.

Birth certificate data also provided maternal age in years, race/ ethnicity (non-Hispanic Caucasian, non-Hispanic AfricanAmerican, non-Hispanic Asian/Pacific Islander, non-Hispanic other race or Hispanic); marital status (married or living with partner; separated, divorced or widowed; or never married); and health insurance (private, any public or no insurance). At 9 months, we assessed socioeconomic status (defined using composite index generated by NCES that incorporated parental education, occupation and household income) and US region of residence.

\section{Analyses}

Descriptive analyses were conducted using survey procedures from SAS (V.9.2, Cary, North Carolina, USA); we generated summary statistics to describe sample characteristics and used $\chi^{2}$ and $\mathrm{t}$-tests to evaluate differences in covariates between women who did and did not experience any PSLE and by children's VLBW status. We then used MPlus (V.7.1, Los Angeles, California, USA) to conduct path analyses using linear probability models (with binary outcomes) that assessed direct and indirect effects of PSLEs and VLBW on: (1) overall child health, (2) SHCN and (3) severe health conditions. This approach enabled us to account for multiple sequential and temporal pathways and to specify and test both direct effects (eg, of PSLEs on infant and toddler health) and indirect effects (eg, of PSLEs on infant and toddler health via birth weight) of PSLEs on the outcomes of interest. ${ }^{20}$ Linear probability path analysis models were used to derive interpretable estimates of direct and indirect effects, specifically so that coefficients represented the percent change in the probability each child health outcome for a 1 unit change in each independent variable, holding everything else constant (eg, the change in the probability of having a child in fair/poor health when the mother was exposed to any PSLE compared with none). Analyses adjusted for appropriate covariates (see figure 1) and accounted for ECLS-B's complex sampling design. 


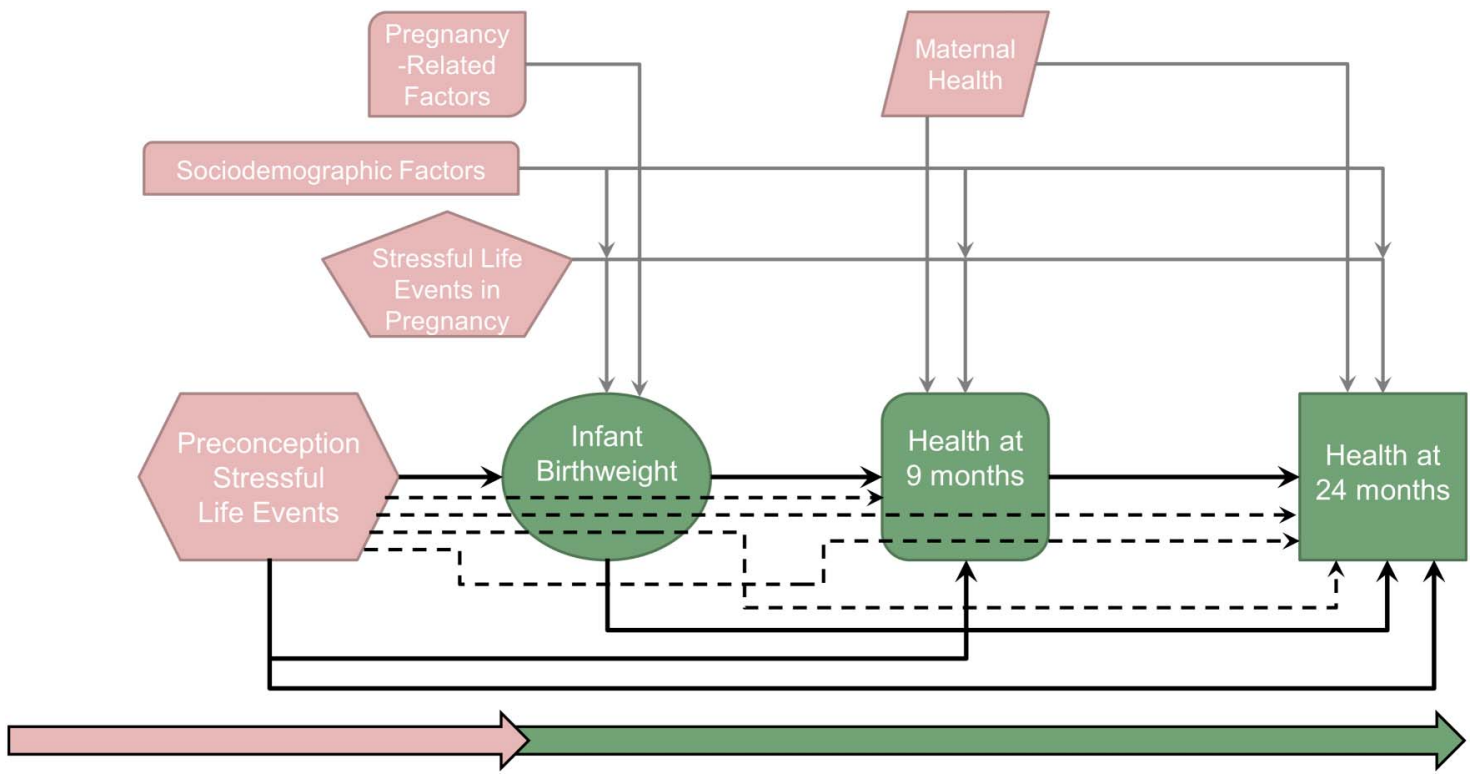

Figure 1 Displays the direct and indirect effects included in the path models. Primary direct effects of interest (depicting relationships among exposure to stressful life events prior to conception, infant birth weight and child health outcomes) are shown with solid black arrows. Direct effects for groups of adjustment variables are shown with solid gray arrows. Maternal factors are depicted in light grey and infant and child outcomes are depicted in darker grey. Variables included as sociodemographic factors are: maternal chronic conditions, number of children born, parity, maternal age, race/ethnicity, marital status, health insurance status, socioeconomic status and region of residence. Variables included as pregnancy-related factors are: pregnancy complications, pre-pregnancy body mass index and initiation of prenatal care. Specific indirect effects are shown as dashed black arrows, and map the hypothesised pathway through intermediate variables.

\section{RESULTS}

Tables 1 and 2 present descriptive statistics and bivariate associations of covariates and child health outcomes to PSLEs and VLBW status. In the entire sample, $19.0 \%$ of women experienced any PSLE; $28.6 \%$ of children who were born VLBW had mothers who experienced any PSLE (table 1). At 9 months, $2.4 \%$ of infants were in fair/poor health, $6.4 \%$ had SHCN, and $7.5 \%$ had severe health conditions (table 2); by 24 months, the percentage of toddlers with SHCN increased to $15.5 \%$. VLBW children were more likely to have poor health, SHCN, and severe health conditions at 9 and 24 months than NBW children (all $\mathrm{p}<0.01$ ).

Table 3 and figure 2 summarise path model coefficients for the direct and indirect effects between PSLEs, VLBW and child health outcomes at 9 and 24 months. Path models for each of the three health outcomes demonstrated good model fit (table 3), suggesting that no important paths had been omitted. As expected, there was a significant direct effect of PSLEs on VLBW, such that women exposed to PSLEs had a higher probability of delivering a VLBW infant. In turn, being born VLBW showed direct effects on all three child health measures at 9 and 24 months, ranging from a $4.1 \%$ increase in the probability of fair/poor health at 24 months to a $26.5 \%$ increase in the probability of SHCN at 9 months. Fair/poor health status, SHCN and severe health conditions at 9 months were significantly associated with increased probability of similar adverse health status at 24 months (fair/poor health $\beta=13.2 \%$, 95\% CI $6.0 \%$ to $20.4 \%$; SHCN $\beta=28.3 \%, 95 \%$ CI $22.4 \%$ to $34.1 \%$; severe health conditions $\beta=20.6 \%, 95 \%$ CI $15.7 \%$ to $25.4 \%)$.

Although PSLEs did not display significant direct effects on any health outcome at 9 or 24 months, small but statistically significant indirect effects were observed such that PSLEs affected the probability of fair/poor health $(\beta=0.03 \%, 95 \%$ CI $0.01 \%$ to
$0.05 \%)$, SHCN $(\beta=0.15 \%, 95 \%$ CI $0.06 \%$ to $0.24 \%)$ and severe health conditions ( $\beta=0.05 \%, 95 \%$ CI $0.01 \%$ to $0.08 \%$ ) at 9 months through the effect of PSLEs on VLBW. While the total indirect effect of PSLEs on child health at 24 months was not significant, the specific indirect effects of PSLEs on child health at 24 months that operated through VLBW were statistically significant (table 3 ).

In sensitivity analyses, neither the direct effect from PSLEs to LBW nor the indirect effects from PSLEs to outcomes at 9 or 24 months through LBW were significant (see online supplementary appendix 1). Comparing VLBW children to all other children weighing $>1500 \mathrm{~g}$ yielded similar results (see online supplementary appendix 2).

\section{DISCUSSION}

In this population-based cohort of US families, we found evidence for a small indirect association between women's exposure to stressful life events in the preconception period and the likelihood of fair or poor health, SHCNs or severe health conditions in offspring. As hypothesised, the effects of PSLEs on these early markers of children's health operated via a 'chains-of-risk' model, ${ }^{6}$ whereby PSLEs increased the risk of VLBW, which, in turn, increased the risk of children's poor health. These findings are consistent with our previous study using the same dataset, which indicated that exposure to PSLEs increases the risk for $\mathrm{VLBW}^{7}$ and with a large body of evidence demonstrating the deleterious effects of VLBW on children's long-term health. ${ }^{8-11}$ The path models identified here suggest that women's exposure to PSLEs may not exert a direct effect on early child health but could, via an indirect pathway, place very young children at risk through the increased risk of being born VLBW. Although the effects were relatively small, our findings highlight the potential importance of women's preconception environment and suggest avenues for future research. 
Table 1 Descriptive statistics by maternal exposure to stressful life events prior to conception and infant birth weight status, national data from the Early Childhood Longitudinal Study-Birth Cohort (ECLS-B), 2001-2003

\begin{tabular}{|c|c|c|c|c|c|c|c|}
\hline & \multirow[b]{2}{*}{ Total } & \multicolumn{3}{|c|}{ Stressful life events prior to conception } & \multicolumn{3}{|c|}{ Infant birth weight status } \\
\hline & & None & Any & $\mathrm{p}$ Value & Very low & Normal & p Value \\
\hline Total (weighted) & 3182840 & 2577421 & 605419 & & 44732 & 3138108 & \\
\hline Per cent & & $81.0 \%$ & $19.0 \%$ & & $1.4 \%$ & $98.6 \%$ & \\
\hline Total (unweighted)* & 6900 & 5450 & 1400 & & 900 & 5950 & \\
\hline Infant birth weight status & & & & $<0.001$ & & & - \\
\hline Very low & $1.4 \%$ & $1.2 \%$ & $2.1 \%$ & & $100.0 \%$ & - & \\
\hline Normal & $98.6 \%$ & $98.8 \%$ & $97.9 \%$ & & - & $100.0 \%$ & \\
\hline Stressful life events prior to conception & & & & - & & & $<0.001$ \\
\hline None & $81.0 \%$ & $100.0 \%$ & - & & $71.4 \%$ & $81.1 \%$ & \\
\hline Any & $19.0 \%$ & - & $100.0 \%$ & & $28.6 \%$ & $18.9 \%$ & \\
\hline \multicolumn{8}{|l|}{ Health, stress and sociodemographic factors } \\
\hline Pregnancy complications & & & & 0.36 & & & $<0.001$ \\
\hline None & $87.4 \%$ & $87.7 \%$ & $86.6 \%$ & & $57.0 \%$ & $87.9 \%$ & \\
\hline Any & $12.6 \%$ & $12.3 \%$ & $13.4 \%$ & & $43.0 \%$ & $12.1 \%$ & \\
\hline Maternal chronic conditions & & & & 0.23 & & & $<0.001$ \\
\hline None & $80.1 \%$ & $80.5 \%$ & $78.5 \%$ & & $60.8 \%$ & $80.4 \%$ & \\
\hline Any & $19.9 \%$ & $19.5 \%$ & $21.5 \%$ & & $39.2 \%$ & $19.6 \%$ & \\
\hline Maternal health status, mean (SD) $\dagger$ & $2.0(0.95)$ & $2.0(0.95)$ & $2.1(0.97)$ & 0.80 & $2.3(3.1)$ & $2.0(0.89)$ & $<0.001$ \\
\hline Pre-pregnancy body mass index & & & & 0.29 & & & 0.01 \\
\hline Normal, underweight or unknown & $56.2 \%$ & $56.5 \%$ & $54.5 \%$ & & $51.2 \%$ & $56.2 \%$ & \\
\hline Overweight or obese & $43.8 \%$ & $43.5 \%$ & $45.5 \%$ & & $48.8 \%$ & $43.8 \%$ & \\
\hline Initiation of prenatal care & & & & 0.22 & & & $<0.001$ \\
\hline In the first trimester & $95.5 \%$ & $95.5 \%$ & $95.3 \%$ & & $93.6 \%$ & $95.5 \%$ & \\
\hline In the second or third trimester & $4.2 \%$ & $4.2 \%$ & $4.0 \%$ & & $4.5 \%$ & $4.2 \%$ & \\
\hline Did not receive prenatal care & $0.3 \%$ & $0.3 \%$ & $0.7 \%$ & & $1.9 \%$ & $0.3 \%$ & \\
\hline Plurality & & & & $<0.001$ & & & $<0.001$ \\
\hline Singleton & $98.8 \%$ & $99.1 \%$ & $97.7 \%$ & & $81.7 \%$ & $99.1 \%$ & \\
\hline Multiple & $1.2 \%$ & $0.9 \%$ & $2.3 \%$ & & $18.3 \%$ & $0.9 \%$ & \\
\hline Parity & & & & $<0.001$ & & & 0.01 \\
\hline None & $41.0 \%$ & $44.8 \%$ & $24.8 \%$ & & $44.9 \%$ & $41.0 \%$ & \\
\hline One or more & $59.0 \%$ & $55.2 \%$ & $75.2 \%$ & & $55.1 \%$ & $59.0 \%$ & \\
\hline Stressful life events during pregnancy & & & & 0.02 & & & 0.66 \\
\hline None & $94.1 \%$ & $93.8 \%$ & $95.6 \%$ & & $93.7 \%$ & $94.2 \%$ & \\
\hline Any & $5.9 \%$ & $6.2 \%$ & $4.4 \%$ & & $6.3 \%$ & $5.8 \%$ & \\
\hline Maternal age, years, mean (SD) & $27.2(6.1)$ & $26.3(5.8)$ & $30.8(6.2)$ & $<0.001$ & $27.1(19.7)$ & $27.2(5.7)$ & 0.85 \\
\hline Maternal race/ethnicity & & & & 0.01 & & & $<0.001$ \\
\hline Non-Hispanic white & $57.0 \%$ & $56.1 \%$ & $61.0 \%$ & & $46.0 \%$ & $57.2 \%$ & \\
\hline Non-Hispanic black & $14.3 \%$ & $14.3 \%$ & $13.9 \%$ & & $27.5 \%$ & $14.1 \%$ & \\
\hline Non-Hispanic Asian/Pacific Islander & $3.6 \%$ & $3.7 \%$ & $3.1 \%$ & & $2.8 \%$ & $3.6 \%$ & \\
\hline Non-Hispanic other race & $2.6 \%$ & $2.5 \%$ & $3.1 \%$ & & $2.1 \%$ & $2.6 \%$ & \\
\hline Hispanic & $22.5 \%$ & $23.3 \%$ & $18.9 \%$ & & $21.7 \%$ & $22.5 \%$ & \\
\hline Marital status & & & & $<0.001$ & & & $<0.001$ \\
\hline Married or living with partner & $83.4 \%$ & $83.1 \%$ & $84.7 \%$ & & $76.7 \%$ & $83.5 \%$ & \\
\hline Separated/divorced/widowed & $3.1 \%$ & $2.5 \%$ & $5.6 \%$ & & $3.1 \%$ & $3.1 \%$ & \\
\hline Never married & $13.5 \%$ & $14.4 \%$ & $9.7 \%$ & & $20.2 \%$ & $13.4 \%$ & \\
\hline Health insurance & & & & 0.10 & & & $<0.001$ \\
\hline Private only & $58.9 \%$ & $58.2 \%$ & $62.0 \%$ & & $51.6 \%$ & $59.0 \%$ & \\
\hline Any public & $37.5 \%$ & $38.3 \%$ & $34.2 \%$ & & $44.6 \%$ & $37.4 \%$ & \\
\hline None & $3.6 \%$ & $3.5 \%$ & $3.8 \%$ & & $3.8 \%$ & $3.5 \%$ & \\
\hline Socioeconomic status, mean (SD)‡ & $-0.08(0.81)$ & $-0.09(0.82)$ & $-0.04(0.80)$ & 0.20 & $-0.25(2.4)$ & $-0.08(0.76)$ & $<0.001$ \\
\hline Region of residence & & & & 0.30 & & & 0.01 \\
\hline Northeast & $17.0 \%$ & $17.2 \%$ & $15.8 \%$ & & $17.7 \%$ & $17.0 \%$ & \\
\hline Midwest & $22.2 \%$ & $22.2 \%$ & $22.0 \%$ & & $21.0 \%$ & $22.2 \%$ & \\
\hline South & $36.9 \%$ & $36.3 \%$ & $39.6 \%$ & & $42.1 \%$ & $36.8 \%$ & \\
\hline West & $24.0 \%$ & $24.3 \%$ & $22.7 \%$ & & $19.2 \%$ & $24.0 \%$ & \\
\hline
\end{tabular}


Table 2 Child health at 9 and 24 months by maternal exposure to stressful life events prior to conception and infant birth weight status, national data from the Early Childhood Longitudinal Study-Birth Cohort (ECLS-B), 2001-2003

\begin{tabular}{|c|c|c|c|c|c|c|c|}
\hline & \multirow[b]{2}{*}{ Total } & \multicolumn{3}{|c|}{ Stressful life events prior to conception } & \multicolumn{3}{|c|}{ Infant birth weight status } \\
\hline & & None & Any & p Value & Very low & Normal & p Value \\
\hline \multicolumn{8}{|c|}{ Child health outcomes } \\
\hline \multicolumn{8}{|c|}{ Fair or poor health status (months) } \\
\hline At 9 & $2.4 \%$ & $2.6 \%$ & $1.4 \%$ & 0.03 & $8.2 \%$ & $2.3 \%$ & $<0.001$ \\
\hline At 24 & $2.0 \%$ & $1.9 \%$ & $2.3 \%$ & 0.51 & $7.6 \%$ & $1.9 \%$ & $<0.001$ \\
\hline \multicolumn{8}{|c|}{ Any special healthcare need (months) } \\
\hline At 9 & $6.4 \%$ & $6.5 \%$ & $6.0 \%$ & 0.58 & $33.5 \%$ & $6.0 \%$ & $<0.001$ \\
\hline At 24 & $15.5 \%$ & $15.6 \%$ & $15.0 \%$ & 0.69 & $45.5 \%$ & $15.0 \%$ & $<0.001$ \\
\hline \multicolumn{8}{|c|}{ Any severe health condition (months) } \\
\hline At 9 & $7.5 \%$ & $7.6 \%$ & $7.3 \%$ & 0.80 & $17.2 \%$ & $7.4 \%$ & $<0.001$ \\
\hline At 24 & $8.1 \%$ & $7.8 \%$ & $9.6 \%$ & 0.16 & $20.2 \%$ & $7.9 \%$ & $<0.001$ \\
\hline
\end{tabular}

$\mathrm{N}=6900$. Weighted estimates. Tables show prevalence estimates for each child health outcome by exposure to stressful life events prior to conception and infant birth weight status. The reference categories are 'excellent, very good or good' health, no special healthcare need, and no severe health condition at each time point.

Preconception stress has been previously examined as a determinant of perinatal outcomes. For example, using data from the ECLS-B, Witt et $\mathrm{al}^{7}$ found that exposure to at least one PSLE increased the odds of VLBW by $38 \%$. In separate studies, the authors also found associations between PSLEs and the likelihoods of PTB among teenage mothers ${ }^{15}$ and of delivering via medically indicated caesarean section. ${ }^{16}$ Similar associations have also been noted in Danish populations. ${ }^{22}$ Fewer studies have examined the prospective effect of preconception stress with outcomes after the perinatal period, although some European-based evidence ties preconception stress to the risk of infant mortality, ${ }^{13}$ childhood ADHD, ${ }^{12}$ overweight status in adolescence $^{12}$ and adult affective disorder in men. ${ }^{23}$ Still, other studies have reported null associations. ${ }^{24-26}$ To our knowledge, the present study is the first to expand on this work by comprehensively testing the relations among maternal exposure to PSLEs, VLBW and early child health in a national cohort of US families.

Several mechanisms may explain our findings. The accumulation of stress across the life course has been theorised to increase allostatic load or 'weathering', leading to a decline in reproductive health. ${ }^{2} 27$ High cortisol levels or immune dysfunction during pregnancy could contribute to neuroendocrine, immune and inflammatory processes that are associated with poor birth outcomes. $^{28} 29$ Women's exposure to PSLEs may also have epigenetic effects in utero that, in turn, increase children's susceptibility to disease. ${ }^{30}$ This hypothesis is supported by research showing heightened levels of total IgE (a biomarker of atopic

Table 3 Path analysis results of the association between maternal exposure to stressful life events prior to conception and offspring overall health, special healthcare needs and severe health conditions in early childhood through very low birth weight

\begin{tabular}{|c|c|c|c|c|c|c|}
\hline & \multicolumn{2}{|c|}{ Fair or poor health } & \multicolumn{2}{|c|}{ Special healthcare needs } & \multicolumn{2}{|c|}{ Severe health conditions } \\
\hline & $\boldsymbol{\beta}$ & $95 \% \mathrm{Cl}$ & $\boldsymbol{\beta}$ & $95 \% \mathrm{Cl}$ & $\boldsymbol{\beta}$ & $95 \% \mathrm{Cl}$ \\
\hline \multicolumn{7}{|l|}{ Direct effects } \\
\hline From PSLEs to VLBW & $0.55 \%$ & (0.23 to 0.87$)$ & $0.55 \%$ & (0.23 to 0.87 ) & $0.55 \%$ & (0.23 to 0.87$)$ \\
\hline From PSLEs to $\mathrm{CH}$ at 9 months & $-0.81 \%$ & $(-1.75$ to 0.14$)$ & $-0.98 \%$ & $(-2.86$ to 0.90$)$ & $1.03 \%$ & $(-1.27$ to 3.34$)$ \\
\hline From PSLEs to $\mathrm{CH}$ at 24 months & $0.56 \%$ & $(-0.48$ to 1.60$)$ & $-0.44 \%$ & (-3.34 to 2.45$)$ & $1.99 \%$ & $(-0.75$ to 4.73$)$ \\
\hline From VLBW to $\mathrm{CH}$ at 9 months & $5.33 \%$ & (2.97 to 7.69 ) & $26.53 \%$ & (22.53 to 30.53 ) & $8.92 \%$ & (5.91 to 11.92 ) \\
\hline From VLBW to $\mathrm{CH}$ at 24 months & $4.10 \%$ & (2.01 to 6.19 ) & $21.50 \%$ & (17.50 to 25.51$)$ & $9.04 \%$ & (5.96 to 12.12$)$ \\
\hline From $\mathrm{CH}$ at 9 months to $\mathrm{CH}$ at 24 months & $13.19 \%$ & (5.96 to 20.42 ) & $28.25 \%$ & (22.40 to 34.10$)$ & $20.55 \%$ & (15.68 to 25.42$)$ \\
\hline \multicolumn{7}{|l|}{ Indirect effects* } \\
\hline PSLEs $\rightarrow$ VLBW $>$ CH at 9 months & $0.03 \%$ & (0.01 to 0.05$)$ & $0.15 \%$ & (0.06 to 0.24$)$ & $0.05 \%$ & (0.01 to 0.08$)$ \\
\hline PSLEs $>$ CH at 24 months & $-0.08 \%$ & $(-0.22$ to 0.06$)$ & $-0.12 \%$ & $(-0.69$ to 0.46$)$ & $0.27 \%$ & $(-0.21$ to 0.75$)$ \\
\hline PSLEs $>\mathrm{CH}$ at 9 months $>\mathrm{CH}$ at 24 months & $-0.11 \%$ & $(-0.25$ to 0.04$)$ & $-0.28 \%$ & $(-0.82$ to 0.26$)$ & $0.21 \%$ & $(-0.27$ to 0.70$)$ \\
\hline PSLEs $>$ VLBW $>$ CH at 24 months & $0.02 \%$ & (0.01 to 0.04 ) & $0.12 \%$ & $(0.04$ to 0.20$)$ & $0.05 \%$ & (0.02 to 0.08$)$ \\
\hline PSLEs $>$ VLBW $>$ CH at 9 months $>\mathrm{CH}$ at 24 months & $0.01 \%$ & (0.00 to 0.01$)$ & $0.04 \%$ & (0.02 to 0.07 ) & $0.01 \%$ & (0.00 to 0.02$)$ \\
\hline \multicolumn{7}{|l|}{ Model fit statistics } \\
\hline$\chi^{2}$ statistic and $p$ value & 14.3 & 0.11 & 15.9 & 0.11 & 11.2 & 0.26 \\
\hline $\mathrm{CFI}$ & 0.991 & & 0.991 & & 0.997 & \\
\hline RMSEA & 0.009 & & 0.011 & & 0.006 & \\
\hline
\end{tabular}


Fair or Poor Health

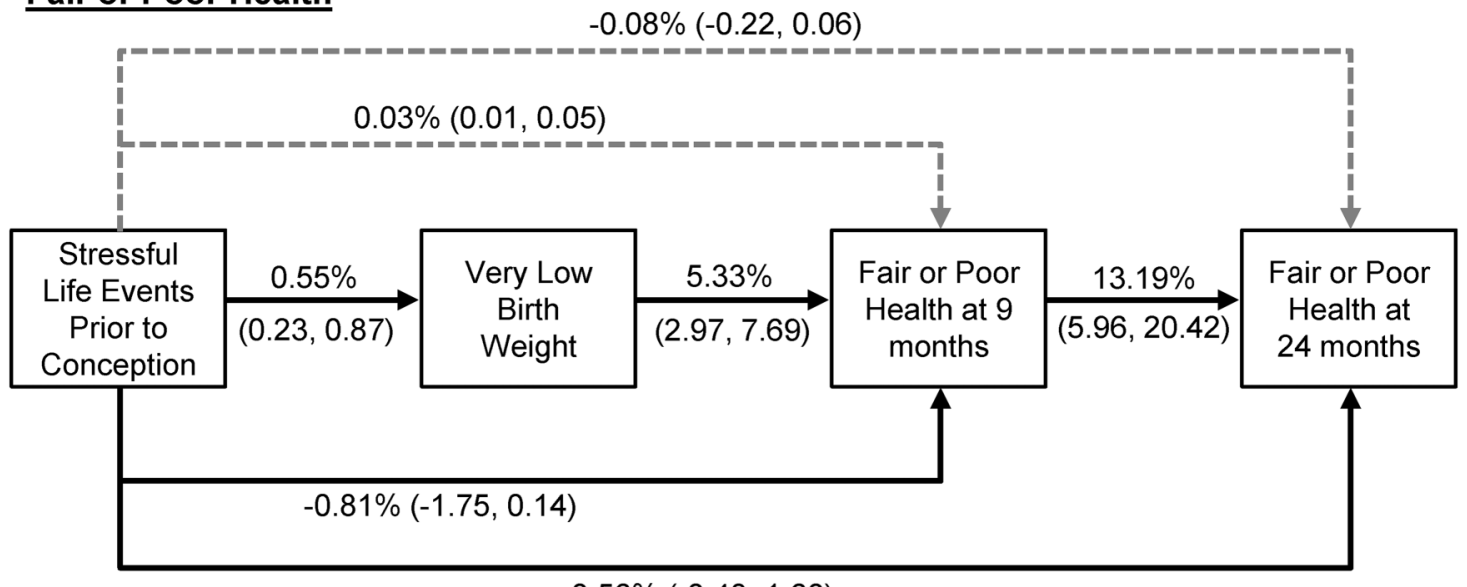

$0.56 \%(-0.48,1.60)$

\section{Special Health Care Needs}

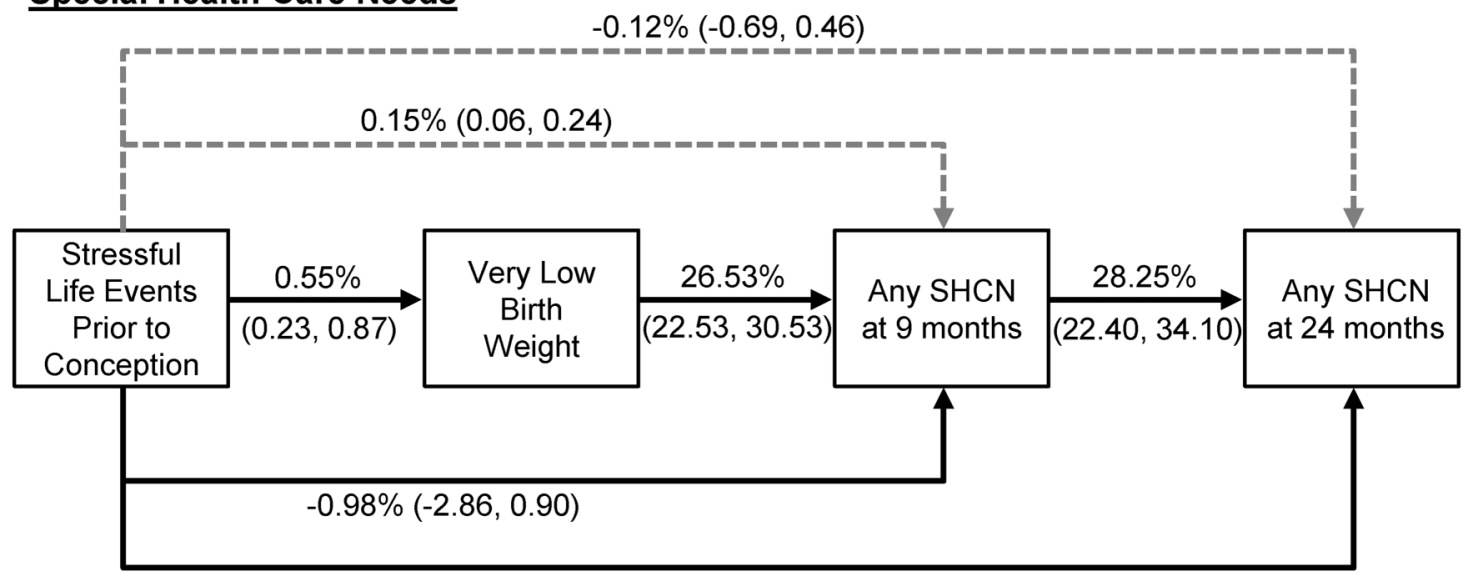

$-0.44 \%(-3.34,2.45)$

\section{Severe Health Conditions}

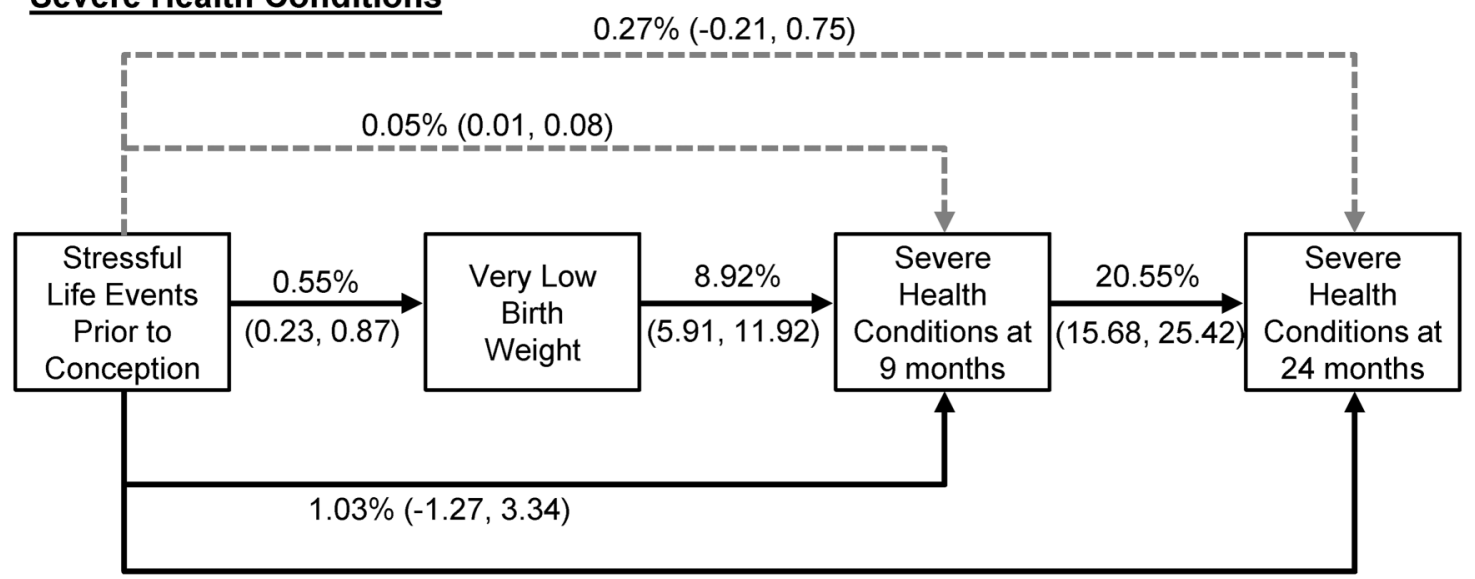

$1.99 \%(-0.75,4.73)$

Figure 2 Summarises the path analyses results of selected direct and total indirect effects of maternal exposure to stressful life events in the preconception period on very low birth weight and child health at 9 and 24 months of age. Weighted results are presented as per cent changes and corresponding $95 \% \mathrm{Cls}$, controlling for pregnancy complications, maternal chronic conditions, pre-pregnancy body mass index, initiation of prenatal care, plurality, parity, exposure to any stressful life event during pregnancy, maternal race/ethnicity, maternal age, marital status at birth, health insurance coverage, family socioeconomic status and US region of residence. Direct effects from very low birth weight status to each child health variable at 24 months are also included. Solid lines represent direct effects and dashed lines indicate total indirect effects (SHCN, special healthcare need). 
risk) in cord blood of children born to mothers exposed to interpersonal trauma. ${ }^{31}$ Alternatively, women exposed to stressors might be more likely to engage in negative health behaviours, such as smoking, a correlate of both infant birth weight ${ }^{32}$ and children's health. ${ }^{33}$ However, we note that the percent differences we found in this study were, in most cases, small, as exposure to PSLEs is likely only one component of a more comprehensive set of intergenerational pathways linking maternal and child health. Other factors may play important roles in these pathways (eg, gestational age, shared environmental risks, maternal stress reactivity) that may spillover to influence the child. Research should identify additional preconception risk factors and determine how they influence social, behavioural and physiological processes that contribute to health outcomes in the next generation, and pinpoint specific critical periods (eg, in utero, neonatal period, etc) in which the effects of PSLEs are most salient. This may help us better understand the biological mechanisms connecting PSLEs and child health and the potential intergenerational transmission of stress.

We note several limitations. Children who died before 9 months of age were not eligible to participate in the ECLS-B, potentially leading to conservative estimates of the effect of PSLEs on birth weight and child health. The ECLS-B retrospectively collected limited data on PSLEs and may not have comprehensively captured the spectrum of stressors women experience. This might have resulted in misclassification. The number of individuals who endorsed specific PSLEs was small, so we were unable to examine the independent effects of each type of event. The null finding of an indirect effect of PSLEs on child health through LBW could be explained by several mechanisms, including misclassification of infant birth weight ${ }^{34} 35$ or undetected dose-response effects. Alternatively, this finding may indicate that the effects of stressors on child health operate only via extreme effects on fetal growth. These hypothesised pathways could be examined in future research. Birth certificates may incorrectly report some information. ${ }^{36}$ Our SCHNs measure may not have included important conditions; conditions were also reported by mothers, potentially resulting in misclassification. Mothers who experienced PSLEs and/or gave birth to VLBW babies may have different expectations for their child's health. Although not available in the ECLS-B, future work validating our findings using medical record data is warranted.

Despite these limitations, our findings suggest that a pathway may exist between PSLEs, VLBW and early indicators of child health, thereby serving as an important first step to understanding an intergenerational link between maternal and child health that has not been previously examined among US populations. Our path analyses allowed for the decomposition of direct and indirect effects of preconception and obstetric factors on early child health, a notable advancement over previous work. Although the effects we found were mostly small and potentially conservative given data limitations, they complement those in other populations by suggesting that women's preconception stress exposure is associated with a slightly elevated prevalence of poor health in offspring. Future work, utilising more complete data from prospectively designed studies, is needed to replicate these findings and elucidate the mechanisms by which these processes might occur.

Current approaches to understanding the link between maternal and child health have traditionally focused on women's health during pregnancy and postpartum, but growing evidence indicates that there may be opportunities to focus research and prevention efforts further upstream. Women experiencing stress before their pregnancies may need more support than is provided in standard care. While PSLEs themselves may not be preventable, making appropriate mental health and social services accessible to vulnerable women could buffer their effect on obstetric outcomes, thus also potentially benefiting the health of their children.

In conclusion, our analyses suggest that women's exposure to PSLEs not only increases the risk for having a VLBW infant, but may also be associated with children's future health. Better understanding the intergenerational risk pathways connecting maternal and child health may enhance efforts to reduce children's health disparities. One strategy-targeting women's preconception health-would not only address women's own unmet needs, but may also indirectly benefit the early health of their children. Future research should explore this hypothesis and investigate the extent to which children's early health outcomes are associated with specific sensitive developmental periods (eg, maternal adverse childhood events, the immediate preconception period or the perinatal period). This may offer insight into aetiological mechanisms leading to these outcomes and help pave the way for preventive interventions. Our findings emphasise that adopting an intergenerational life course approach may aid such efforts.

\section{What is already known on this subject}

Emerging evidence suggests that women's exposure to stressful life events prior to conception (PSLEs) may affect the immediate and long-term health of offspring. Such evidence supports a life course approach to understanding health and development. No study has examined the relationship between PSLEs and obstetric and child health outcomes in a nationally representative sample of US mothers and their children.

\section{What this study adds}

This study provides an empirical application of the life course approach using nationally representative longitudinal data from the USA. Using path analyses, we show that women's exposure to PSLEs may not exert a direct effect on early child health but, via an indirect pathway, place very young children at risk through the increased risk of being born very low birth weight.

Contributors ERC helped conceptualise and design the study, interpreted the data, drafted the initial manuscript, and approved the final manuscript as submitted. HP assisted with the study design, conducted the final analyses, reviewed and revised the manuscript, and approved the final manuscript as submitted. LEW helped conceptualise and design the study, conducted preliminary analyses, interpreted the data, critically reviewed and revised the manuscript, and approved the final manuscript as submitted. KCM assisted with the study design, conducted preliminary analyses, reviewed and revised the manuscript, and approved the final manuscript as submitted. FW, KL and DC helped interpret the data, reviewed and revised the manuscript, and approved the final manuscript as submitted. WPW conceptualised and designed the study, was responsible for the acquisition of data, critically reviewed and revised the manuscript, and approved the final manuscript as submitted.

Funding This project was made possible by a Health Resources and Services Administrative (HRSA) (W. P.Witt, L. E. Wisk, and D. Chatterjee: R40MC23625; principal in- vestigator W. P. Witt) grant. Additional funding for this research was provided by grants from the Agency for Healthcare Research and Quality (K. Mandell 
T32 HS00083; principal investigator M. Smith), and a National Research Service Award institutional training grant (E. R. Cheng: T32-HD075727; principal investigator J. A. Finkelstein). L. E. Wisk was supported by the Thomas O. Pyle Fellowship and an Agency for Healthcare Research and Quality Postdoctoral Training Grant T32HS000063-20 (principal investigator J. A. Finkelstein). D. Chatterjee was additionally supported by National Research Service Award (NRSA) in Primary Medical Care Grant T32HP22239 (principal investigator: I. Borowsky). US Department of Health and Humanl Services >Agency for Healthcare Research and Quality. T32HS000063-20. T32HS00083; US Department of Health and Human Services $>$ Health Resources and Services Administration. R40MC23625.

T32HP22239; US Department of Health and Human Services > National Institutes of Health > National Institute of Child Health and Human Development.

T32HD075727-01.

Competing interests None declared.

Provenance and peer review Not commissioned; externally peer reviewed.

\section{REFERENCES}

1 Houtrow AJ, Larson K, Olson LM, et al. Changing trends of childhood disability, 2001-2011. Pediatrics 2014;134:530-8.

2 Lu MC, Halfon N. Racial and ethnic disparities in birth outcomes: a life-course perspective. Matern Child Health J 2003;7:13-30.

3 Halfon N, Hochstein M. Life course health development: an integrated framework for developing health, policy, and research. Milbank Q 2002;80:433-79.

4 Elder GH Jr. The life course as developmental theory. Child Dev 1998;69:1-12.

5 Pickles A, De Stavola B. An overview of models and methods for life course analysis. In: Pickles A, Maughan B, Wadsworth M, eds. Epidemiological methods in life course research. Oxford: Oxford University Press, 2007:181-220.

6 Kuh D, Ben-Shlomo Y, Lynch J, et al. Life course epidemiology. J Epidemiol Community Health 2003;57:778.

7 Witt WP, Cheng ER, Wisk LE, et al. Maternal stressful life events prior to conception and the impact on infant birth weight in the United States. Am J Public Health 2014; 104:S81-9

8 Heron M. Deaths: leading causes for 2008. Natl Vital Stat Rep 2012;60:1-94.

9 Bhushan V, Paneth N, Kiely JL. Impact of improved survival of very low birth weight infants on recent secular trends in the prevalence of cerebral palsy. Pediatrics 1993;91:1094-100.

10 McCormick MC. The contribution of low birth weight to infant mortality and childhood morbidity. N Engl J Med 1985;312:82-90.

11 Vohr BR, Wright LL, Dusick AM, et al. Neurodevelopmental and functional outcomes of extremely low birth weight infants in the National Institute of Child Health and Human Development Neonatal Research Network, 1993-1994. Pediatrics 2000;105:1216-26.

12 Li J, Olsen J, Vestergaard M, et al. Prenatal stress exposure related to maternal bereavement and risk of childhood overweight. PLOS ONE 2010;5:e11896.

13 Class QA, Khashan AS, Lichtenstein P, et al. Maternal stress and infant mortality: the importance of the preconception period. Psychol Sci 2013;24:1309-16.

14 Bethel J, Green JL, Kalton G, et al. Early Childhood Longitudinal Study, Birth Cohort (ECLS-B), the ECLS-B Methodology Report for the 9-Month Data Collection, 200102 (NCES 2005-147). U.S. Department of Education. Washington, DC: National Center for Education Statistics, 2005.

15 Witt WP, Cheng ER, et al. Preterm birth in the United States: the impact of stressful life events prior to conception and maternal age. Am J Public Health 2014;104 (Suppl 1):S73-80.
16 Witt WP, Wisk LE, Cheng ER, et al. Determinants of cesarean delivery in the US: a lifecourse approach. Matern Child Health I 2015;19:84-93.

17 Eugster A, Vingerhoets A. Psychological aspects of in vitro fertilization: a review. Soc Sci Med 1999:48:575-89.

18 Holmes TH, Rahe RH. The social readjustment rating scale. J Psychosom Res 1967:11:213-18

19 Rahe CRH, Arthur DRJ. Life change and illness studies: past history and future directions. J Human Stress 1978;4:3-15.

20 Rahe RH, Veach TL, Tolles RL, et al. The stress and coping inventory: an educational and research instrument. Stress Med 2000;16:199-208.

21 Hayes AF. Introduction to mediation, moderation, and conditional process analysis: a regression-based approach. New York, NY: Guilford Press, 2013.

22 Khashan AS, McNamee R, Abel KM, et al. Reduced infant birthweight consequent upon maternal exposure to severe life events. Psychosom Med 2008;70:688-94.

23 Khashan AS, McNamee R, Henriksen TB, et al. Risk of affective disorders following prenatal exposure to severe life events: a Danish population-based cohort study. J Psychiatr Res 2011;45:879-85.

24 Class QA, Abel KM, Khashan AS, et al. Offspring psychopathology following preconception, prenatal and postnatal maternal bereavement stress. Psychol Med 2014;44:71-84.

25 Li J, Vestergaard M, Obel C, et al. A nationwide study on the risk of autism after prenatal stress exposure to maternal bereavement. Pediatrics 2009;123:1102-7.

26 Zhu JL, Olsen J, Sorensen HT, et al. Prenatal maternal bereavement and congenital heart defects in offspring: a registry-based study. Pediatrics 2013;131: e1225-30.

27 Geronimus AT. Black/white differences in the relationship of maternal age to birthweight: a population-based test of the weathering hypothesis. Soc Sci Med 1996;42:589-97.

28 Dunkel Schetter C. Psychological science on pregnancy: stress processes, biopsychosocial models, and emerging research issues. Annu Rev Psychol 2011;62:531-58

29 Wadhwa PD, Culhane JF, Rauh V, et al. Stress and preterm birth: neuroendocrine, immune/inflammatory, and vascular mechanisms. Matern Child Health J 2001;5:119-25

30 Wadhwa PD, Buss C, Entringer S, et al. Developmental origins of health and disease: brief history of the approach and current focus on epigenetic mechanisms. Semin Reprod Med 2009;27:358-68.

31 Sternthal MJ, Enlow MB, Cohen S, et al. Maternal interpersonal trauma and cord blood IgE levels in an inner-city cohort: a life-course perspective. J Allergy Clin Immunol 2009;124:954-60.

32 Valero De Bernabe J, Soriano T, Albaladejo R, et al. Risk factors for low birth weight: a review. Eur J Obstet Gynecol Reprod Biol 2004;116:3-15.

33 DiFranza JR, Aligne CA, Weitzman M. Prenatal and postnatal environmental tobacco smoke exposure and children's health. Pediatrics 2004;113:1007-15.

34 Emmerson AJ, Roberts SA. Rounding of birth weights in a neonatal intensive care unit over 20 years: an analysis of a large cohort study. BMJ Open 2013;3: e003650.

35 Edouard L, Senthilselvan A. Observer error and birthweight: digit preference in recording. Public Health 1997;111:77-9.

36 Martin J, Hamilton B, Ventura S, et al. Births: final data for 2010. National Vital Statistics Reports; vol 61 no 1. Hyattsville, MD: National Center for Health Statistics, 2012. 